\title{
Inverter Device
}

National Cancer Institute

\section{Source}

National Cancer Institute. Inverter Device. NCI Thesaurus. Code C50010.

An electrical device that converts direct current to alternating current. 